\title{
Coinfection with Staphylococcus aureus community- acquired pneumonia and mycobacterium turberculosis in a diabetic patient: a case report
}

\begin{abstract}
Pulmonary tuberculosis is a common reason for hospital admission worldwide. The objective of this case report is to demonstrate the importance of the active search for M. tuberculosis along with Community-Acquired Pneumonia (CAP). The 36-yearold male patient, known as diabetic, hypertensive and obese, was admitted to the emergency department of the San Carlos Hospital with bronchopneumonia, mild respiratory distress and simple diabetic decompensation. Based on the radiological findings, the patient is treated with a presumptive diagnosis of a right basal CAP and pulmonary tuberculosis. The first smear analyzed by smear microscopy was found positive by exact number of ARAB (alcohol-resistant acid bacilli).To confirm the diagnosis of pulmonary tuberculosis, the molecular method GeneXpert ${ }^{\circledR M T B}$ / RIF was used, finding a low load without resistance to Rifampicin.
\end{abstract}

Through the analysis of this clinical case, we can reinforce the need to continue with the active search for M. tuberculosis even in acute pneumonias, since mixed infections are more common than is generally believed.

Keywords: community-acquired pneumonia (cap), staphylococcus aureus, mycobacterium turberculosis, coinfection
Volume 3 Issue 3 - 2018

\author{
Ana Gabriela Cruz Chavarría,' Laura Inés \\ Blanco Quirós ${ }^{2}$ \\ 'Chief third shift, San Carlos Hospital, Costa Rica \\ ${ }^{2} \mathrm{Head}$ of Bacteriology Section, San Carlos Hospital, Costa Rica
}

Correspondence: Laura Inés Blanco Quirós, Head of Bacteriology Section, San Carlos Hospital, Alajuela, Costa Rica, Microbiologist and Clinical Chemistry, Specialist in Medical Bacteriology at the University of Costa Rica, Email Iblancoq@gmail.com

Received: May 22, 2018 | Published: June 25, 2018

\section{Introduction}

Mycobacterium turberculosis infects one third of the world's population, with 8.7 million new cases reported annually. ${ }^{1}$ Pulmonary tuberculosis is a common reason for admission to hospital in the all world. The presentation of patients with pulmonary tuberculosis often identical with community-acquired pneumonia (CAP). ${ }^{2}$ Shadock mentions that about 40 percent of pulmonary tuberculosis courses with community-acquired pneumonias. ${ }^{2}$

WHO has worked hard to treat tuberculosis, however the extensive use of floruquinolones has led to selective pressure generating ultraresistant or extensively resistant strains that by 2013 had been described in at least 84 countries. ${ }^{1}$

The objective of this case report is to demonstrate the importance of the active search of M.tuberculosis along with the CAP, its agile diagnosis through molecular methods and the contribution that these techniques represent in the fight for eradication, in developing countries.

\section{Materials and methods}

Male patient, 36 year-old, known as diabetic, hypertensive and obese with a history of cessation of smoking 4 years ago, heavy machinery driver, enters the emergency service of the San Carlos Hospital with a bronchopneumonia, mild respiratory distress and a simple diabetic decompensation.

The patient reports having been treated on March 15th, 2018 at the Health Center of Aguas Zarcas where he was administered corticosteroids (Dexamethasone), a $\beta_{2}$ adrenergic agonist as a bronchodilator (inhaled Salbutamol) and antibiotic therapy based on Macrolides (Azithromycin)

Upon his admission on March 19th to the emergency room, the patient was conscious, oriented, without fever and mild respiratory distress. The results of the Arterial Gases were as follows: $\mathrm{pH} 7.48 \mathrm{PO}_{2}$ $60.5 \mathrm{~mm} / \mathrm{Hg}$ Lactate $1.4 \mathrm{mmol} / \mathrm{L} \mathrm{PCO} 33.2 \mathrm{~mm} / \mathrm{Hg} \mathrm{BE}-1.3 \mathrm{mmol} / \mathrm{L}^{2}$ $\mathrm{HCO}^{3}-25.4 \mathrm{mmol} / \mathrm{L}$ Glucose $374 \mathrm{mg} / \mathrm{dL}$ generating a decompensated respiratory alkalosis.

The chest X-ray that we observed in Figure 1 showed a right paracardiac consolidation and a left paracardiac image similar to a cavern or a consolidation in formation, without pleural effusion and with a normal cardiac silhouette.

The analysis of the sputum by piogens, smear microscopy, molecular methods and culture by mycobacteria is carried out. Additionally; as part of the differential diagnosis, a nasopharyngeal swab is performed, which is analyzed by molecular methods to detect respiratory viruses. Based on the radiological findings, the patient is treated with a presumptive diagnosis of a right basal CAP and pulmonary tuberculosis.

In the culture by piogens, two microorganisms are isolated: Staphylococcus aureus in an abundant growth and Klebsiella pneumoniae in a scanty growth, considering S.aureus as the causal agent of the CAP based on the correlation with the clinical manifestations and continuing the antibiotic therapy that it had begun empirically.

On the other hand, the analysis of respiratory virus by molecular methods was negative for Sincitial Respiratory Virus and Influenza $\mathrm{A}$ and $\mathrm{B}$.

The first sputum analyzed by bacilloscopy was found positive by exact number of ARAB (alcohol-resistant acid bacilli) so that we proceed to detect M.tuberculosis by the molecular method GeneXpert ${ }^{\circledR} M T B$ / RIF finding a low load without resistance to Rifampicin.

Once the diagnosis of pulmonary tuberculosis is confirmed, the patient is hospitalized in an area of strict isolation, the therapy with 
specific antibiotics against $M$. tuberculosis of the first line begins and the stabilization of the patient is completed.

The patient remains hospitalized until the full resolution of CAP and the compensation of their chronic diseases, being released on March 30th to continue the supervised antifimic therapy through the primary health services.

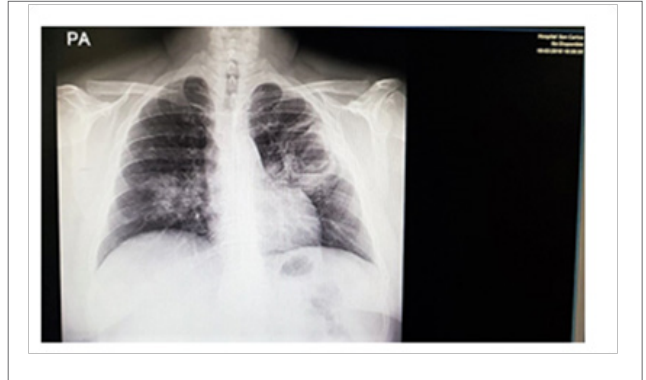

Figure I Pulmonary tuberculosis cavitated.

\section{Discussion}

Although pulmonary tuberculosis is known as a chronic disease, it can be presented as an acute pneumonia. The differentiation between acute tuberculous and non-tuberculous pneumonia is a major challenge in endemic areas. $^{3}$

In their study, Naderi and company found that patients with tuberculous pneumonia had a significantly higher frequency of weight loss, anemia, absence of fever on admission and a longer duration of symptoms ${ }^{3}$ which coincides with this case. It is very important to consider that his chronic diseases compromise him immunologically so he is a candidate for acute multimicrobial infections and the therapeutic failure of macrolide-based therapy is another indication to be taken into account.

On the other hand, the chest X-ray was decisive in the presumptive diagnosis of pulmonary tuberculosis, however; radiographically, the differentiation between active and inactive disease can only be made according to the evolution over time. ${ }^{4}$

Castiñeira mentions that the typical radiological aspect of an acute pulmonary tuberculosis is of air spaces characterized by a homogeneous opacity of ill-defined edges. It is often indistinguishable from typical bacterial pneumonia. Important keys for diagnosis are associated lymphadenopathy (the presence of parenchymal disease in the absence of adenopathy appears only in $1 \%$ of pediatric cases and in $38-81 \%$ of adult primary TB), the lack of systemic toxicity (tuberculous pneumonia can be seen as a casual finding in an asymptomatic patient) and failure in the response to conventional antibacterial therapy. Cavitation is a rare manifestation of primary tuberculosis in infants and children living in communities long exposed to the tubercle bacillus. It has been found in $7-29 \%$ of adults and occurs in $40-87 \%$ of patients with post-primary tuberculosis. ${ }^{4}$

The GeneXpert ${ }^{\circledR}$ MTB / RIF integrates sputum processing, DNA extraction and amplification in a single step. This test enables the detection of resistance to rifampicin through the detection of mutations in the rpo B gene. ${ }^{5}$

In their review, Teran \& de Waard comment on the accuracy of this method compared to microscopy, where the Xpert巴 ${ }^{\circledR}$ MTB / RIF increases the detection of TB among cases confirmed by culture by $23 \%$ while for the detection of resistance to rifampin, the Xpert ${ }^{\circledR}$
MTB / RIF shows an accumulated sensitivity of $95 \%$ and acumulative specificity of $98 \%{ }^{5}$

So that the use of this methodology was decisive in the management of this patient, speeding up the start of antifimic therapy, minimizing the stay in the emergency room and in turn the total hospital stay.

\section{Conclusion}

Through the analysis of this clinical case we can reinforce the need to continue with the active search for M.tuberculosis even in acute pneumonias, since as we discussed above, mixed infections are more common than is generally believed.

In endemic areas such as ours, the joint work of the clinician, the radiology staff and the microbiologist is crucial to address all cases of pulmonary and extrapulmonary tuberculosis, in order to reduce transmission, contributing to the great goal of eradicating the disease Tuberculosis.

\section{Acknowledgments}

None.

\section{Conflict of interest}

The Author declares no conflict of interests.

\section{References}

1. Mandell, Douglas, Bennett's. Enfermedades Infecciosas. Principios $y$ Práctica. 2016;8.

2. Shaddock E, Bosman N, Nana T, et al. Secondary bacterial infection in active pulmonary tuberculosis. Southern African Journal of Infectious Diseases. 2014;29(1):23-26.

3. Naderi H, Sheybani F, Erfani SS. The mask of acute bacterial pneumonia may disguise the face of tuberculosis. Electronic Physician 2017;9(3):3943-3949.

4. Estéveza AC, López PMR., Pena RMJ. Manifestaciones radiológicas de la tuberculosis pulmonar. Medicina Integral. 2002;39(5):192-206.

5. Rommy Teran, Jacobus H. Recientes avances en el diagnóstico de tuberculosis en el laboratorio clínico. The Journal of International Federetion of Clinical Chemistry and Laboratory Medicine. 2015;26(4):310-325. 